parameters identified a high proportion of patients with subclinical evidence of liver injury. Supervised detoxification was completed in 170 patients.

Conclusion Alcohol related hospital admissions can be rapidly identified and managed by a dedicated specialist nursing team working in conjunction with hepatologists and acute physicians. Our cohort demonstrated strong links between poor socioeconomic status and early age of exposure to alcohol, age of regular alcohol misuse and long-term excessive drinking. We have now instituted formal screening of all hospital admissions in ED and MAU with direct electronic referral to the liaison service where alcohol misuse is a factor.

\section{P27 REDUCTION IN ALANINE AMINOTRANSFERASE VALUES IN DIABETIC PATIENTS RECEIVING LONG-TERM ORAL ANTIBIOTICS: DOES MODULATION OF GUT FLORA AFFECT HEPATIC INFLAMMATION IN NON-ALCOHOLIC FATTY LIVER DISEASE?}

doi:10.1136/gutjnl-2011-300857a.27

'L Leach, ' J F L Cobbold, ' J Valabhji, ${ }^{1} \mathrm{M}$ R Thursz. ${ }^{1}$ Hepatology and Gastroenterology section, Department of Medicine, St Mary's Hospital, Imperial College London; ${ }^{2}$ Department of Diabetes, St Mary's Hospital, Imperial College Healthcare NHS Trust

Introduction The gut microbiota may play a role in host metabolic processes and have been linked to the pathophysiology of nonalcoholic fatty liver disease (NAFLD). Murine studies have suggested beneficial effects of modulating the gut microbiota using antibiotics, however studies have not looked at the effect of long-term antibiotic therapy in humans.

Aim To investigate the effects of prolonged broad-spectrum antibiotics in patients attending the diabetic foot clinic who are likely to have NAFLD. It was hypothesised that long-term oral antibiotic administration would result in a decrease in serum alanine aminotransferase (ALT) values after 3 months compared to baseline. Method 43 diabetic patients without known hepatic comorbidity, who had taken a minimum of 3 months' broad-spectrum oral antibiotics (predominantly co-amoxiclav) in the diabetic foot clinic were identified retrospectively. Demographic and clinical data were extracted from clinic letters, patient notes, and computer databases. The primary outcome measure was the change in ALT value at day 90 of antibiotics compared to day 0 . Other outcome measures were a change in patient weight, glycosylated haemoglobin (HbA1c), white cell count, bilirubin and alkaline phosphatase at day 90 from day 0 . The wilcoxon signed rank test was used for paired non-parametric data.

Results All patients with abnormal ALT at baseline $(n=6)$ had a significant decrease in ALT from median $43(41-48) \mathrm{U} / \mathrm{l}$ on day 0 , to $32(29-35) \mathrm{U} / 1$ on day $90(\mathrm{p}=0.03) .37$ patients who had a normal ALT at baseline had a small but significant increase in ALT from median $17(13-23) \mathrm{U} / 1$ on day 0 , to $20(14-28) \mathrm{U} / \mathrm{l}$ on day 90 $(p=0.02)$. In the 20 patients with paired HbA1c values, there was a statistically significant decrease in $\mathrm{HbA1c}$ with antibiotic use $(p=0.03)$. There were no significant associations in change in ALT at 90 days with weight change, white cell count, bilirubin, alkaline phosphatase, antibiotic regime or other medications.

Conclusion The robust decrease in ALT values in patients with abnormal baseline ALTs (and therefore likely to have NAFLD) provides indirect evidence that modulation of gut microbiota with oral antibiotics may reduce hepatic inflammation in NAFLD. The possibility of sepsis-induced liver damage accounting for the changes is countered by no change in white cell count, bilirubin or alkaline phosphatase, while reduction in HbA1c without weight change points to reduced insulin resistance. Prospective studies on patients with biopsy-proven non-alcoholic steatohepatitis are awaited.

\section{P28 DEFECTIVE T-REGULATORY FUNCTION IN AUTOIMMUNE HEPATITIS MAY PARTIALLY DERIVE FROM A PRO- INFLAMMATORY SKEWING OF GAL9+T-REGS}

doi:10.1136/gutjnl-2011-300857a.28

R Liberal, G Mieli-Vergani, D Vergani, M S Longhi. King's College London School of Medicine

Introduction In autoimmune hepatitis (AIH) $\mathrm{CD} 4{ }^{+} \mathrm{CD} 25^{+}$regulatory T-cells (T-regs) are defective in their ability to control CD4 T-cell effector function. T-regs express Galectin9 (Ga19), a $\beta$-galactosidase-binding-protein that inhibits Th1-mediated immuneresponses by binding the T-cell-immunoglobulin-and-mucindomain3 (Tim-3) on CD4 effector cells. In AIH T-regs express reduced levels of Gal9.

Aim To characterise lineage-specific transcription factor and cytokine profiles of peripheral-blood-derived $\mathrm{Gal}^{+} \mathrm{T}$-regs in $\mathrm{AIH}$.

Method 34 ANA/SMA+ patients $(24$ females; median age: 14.6 years) and 17 healthy subjects (HS, 12 females, median age: 29 years) were studied. The phenotype of circulating T-regs was determined by cytofluorimetry using CD4, CD25 and CD127 monoclonal antibodies. The frequency of cells positive for Gal9, FOXP3, T-bet, GATA3 and RORC and that of cells producing IFN $\gamma$, IL-10, TGF- $\beta$ and IL-17 were determined by intracellular staining. Treg suppressor function was evaluated in a proliferation assay following co-culture with CD25 ${ }^{-}$Tim $-3^{+}$and CD25 ${ }^{-}$Tim- $-3^{-}$ autologous target cells.

Results Within $\mathrm{Gal}^{+}$cells the frequency of: (1) $\mathrm{FOXP3}^{+}$cells was lower in AIH than in HS (14.4 \pm 2 vs 42.8 $\pm 3.1, \mathrm{p}<0.001)$; (2) T-bet ${ }^{+}$, GATA3 $^{+}$and RORC ${ }^{+}$cells was similar in AIH and HS; (3) IL-10producing cells was lower in AIH than in HS $(5.1 \pm 0.6$ vs $9.1 \pm 0.5$, $\mathrm{p}<0.001$ ) but higher than in the Ga19- ${ }^{-}$-reg fraction for both (AIH: $5.1 \pm 0.6$ vs $2.3 \pm 0.2, p=0.001$; HS: $9.1 \pm 0.5$ vs $3.3 \pm 0.4, p<0.001)$; (4) TGF- $\beta$-producing cells was lower in AIH than in HS $(6.4 \pm 0.7$ vs $8.1 \pm 0.4, p=0.04)$; (5) IFN $\gamma$ - and IL-17-producing cells was higher in AIH than in HS (IFN $\gamma: 4.4 \pm 0.6$ vs $2.1 \pm 0.3, p=0.002$; IL-17: $4.1 \pm 0.6$ vs $1.8 \pm 0.2, \mathrm{p}=0.002)$. Treatment with anti-IL-10 neutralising antibodies reduced T-reg ability to suppress $\mathrm{CD} 25^{-} \mathrm{Tim}-3^{+}$cell proliferation (AIH: $42 \%$ inhibition in the absence of antibodies vs $36 \%$ in their presence, $p=0.06$; HS: $56 \%$ vs $48 \%, p=0.04$ ), while did not affect $\mathrm{CD} 25^{-}$Tim $-3^{-}$cell proliferation.

Conclusion A skewing towards a pro-inflammatory phenotype and a reduced proportion of $\mathrm{FOXP3}^{+}$and IL-10-producing cells within $\mathrm{Gal9}^{+}$T-regs may contribute to defective immunoregulation in $\mathrm{AIH}$ The reduction of $\mathrm{Gal9}^{+} \mathrm{T}$-reg suppression following anti-IL-10 blockade both in health and AIH, suggests a role for IL-10 in Gal9 ${ }^{+} \mathrm{T}$-cell immune-regulatory function.

\section{P29 REDUCED EXPRESSION OF TIM-3 RENDERS TH1 AND TH17 EFFECTOR CELLS LESS AMENABLE TO T-REG CONTROL IN AUTOIMIMUNE HEPATITIS}

doi:10.1136/gutjnl-2011-300857a.29

R Liberal, G Mieli-Vergani, D Vergani, M S Longhi. King's College London School of Medicine

Introduction In autoimmune hepatitis (AIH), CD4 effector immune responses are permitted by defective $\mathrm{CD} 4{ }^{+} \mathrm{CD} 25^{+}$regulatory $\mathrm{T}$-cells (T-regs). In murine studies apoptosis of Th1 effector cells is mediated by binding of $\mathrm{T}$-cell-immunoglobulin-and-mucin-domain3 (Tim-3) on their surface to Galectin-9 (Ga19) expressed by T-regs. In $\mathrm{AIH}, \mathrm{Tim}-3$ is down-modulated on CD4 effector cells.

Aim To test the frequency of Tim $-3^{+}$cells within the Th1, Th2 and Th17 subsets and to evaluate whether Tim-3 expression by CD4 effectors affects their responsiveness to T-reg control. 